\title{
Just Math: A New Epistemic Frame
}

\author{
Steven F. Wolf*, Leanne Doughty ${ }^{\dagger}$, Paul W. Irving ${ }^{\dagger, *}$, Eleanor C. Sayre $^{* *}$ and \\ Marcos D. Caballero ${ }^{\dagger, *}$ \\ ${ }^{*}$ CREATE for STEM Institute, Michigan State University, East Lansing, MI 48824, USA \\ ${ }^{\dagger}$ Department of Physics and Astronomy, Michigan State University, East Lansing, MI 48824, USA \\ ${ }^{* *}$ Department of Physics, Kansas State University, Manhattan, KS 66506, USA
}

\begin{abstract}
A goal of PER is to understand how students use math in physics contexts. To investigate how students use math, we need to identify transitions between conceptual sense-making about physical systems and using mathematics to describe and to make predictions about those systems. We reviewed video of students solving a variety of physics problems in small groups through the lens of epistemic frames (e-frames). In this paper, we present a new e-frame, which we are calling "Just Math", that is similar to the Worksheet e-frame, but is characterized by brief, low-level, math-focused utterances between students, in some cases along with expansive off-topic discussions. Future work will focus on analyzing the transitions into and out of this e-frame so that we may develop a more coherent understanding of students' use of math in physics.
\end{abstract}

Keywords: Research in physics education, Learning theory and science teaching

PACS: 01.40.Fk, 01.40.Ha

\section{INTRODUCTION}

Physics provides a rich environment to investigate how students use mathematics in science and engineering contexts. Many of the models developed in physics are quantitative and require students to use sophisticated mathematics (e.g., vector calculus) to develop a deep understanding of those models. Furthermore, physics courses are often the gateway to using math in the way that science and engineering professionals do. It is this skillful use of mathematics within physics courses that is a large portion of the challenge for STEM majors.

Research on student use of mathematics in physics frequently focuses on specific physics or mathematical tools (e.g., $[1,2])$. By contrast, other work seeks to develop more general constructs for understanding this vital aspect of students' development (e.g., $[3,4,5,6]$ ), often focusing on describing students' in-the-moment reasoning, not the mathematical tools themselves.

Our work grows out of this latter tradition. In the course of analyzing video of upper-division students solving problems in situ, we noticed that they shift between sense-making activities and activities whose sole purpose appears to be "doing math." We are interested in investigating: how do students collectively set up the problem and determine that they are ready to "just do math"? how (if at all) do students reflect upon and interpret the result they get from doing the math? To answer these questions we need to be able to reliably identify when students are "just doing math." To this end, we have conducted a preliminary study where we leveraged the epistemic frame construct $[7,8]$. We have identified markers in students' behaviors and discourse that are in- dicative of students "just doing math."

In this paper, we describe the features of this new epistemic frame (e-frame), Just Math. We distinguish Just Math from a related e-frame and discuss how this distinction has important implications for our future work.

\section{DATA SOURCE AND CONTEXT}

Our data comes from naturalistic observations of homework help sessions (HHS) in an upper-division electricity and magnetism course. The HHS were an informal weekly meeting where students could work with their colleagues to solve homework problems. The HHS were facilitated by two teaching assistants (TAs) who guided discussion but did not present problems or their solutions. Typically between 5 and 10 students (split into two cooperative groups) attended the session; a core group of 5 students attended regularly. Two pairs of two unattended video cameras recorded each group. The first camera recorded from a distance to capture gestures while the second camera recorded from a high angle above the table to capture students writing on a tablebased whiteboard. In total 114 hours of video was captured. The data presented in this paper is focused on 9 hours of HHS data encompassing 3 different groups of students.

\section{THEORETICAL FRAMEWORK}

Framing is the combination of several resources in an effort to understand and work within a situation [5]. Epis- 
temic framing differs as it is a perception (unconscious or conscious) of the tools and skills required in a particular context or situation. E-frames can be envisioned as a storage area for conceptual and procedural resources, promoting some resource's activation and blocking others [8]. We have drawn from the literature on e-frames because we believe that when students are doing Just Math, they perceive the activity as requiring a particular set of behaviors and math oriented resources.

Building on the work of Scherr and Hammer, we studied the behavioral patterns that students exhibit when working in group settings. In an introductory tutorial setting, Scherr and Hammer identified four e-frames (the Discussion e-frame, the TA e-frame, the Joking e-frame and the Worksheet e-frame) that they characterized by coding for specific behaviors and expectations [7]. For example, in the Worksheet e-frame, students focused their gaze on their personal worksheet, and they hunched their bodies over their paper. Student talk came in short bursts and was monotone. Scherr and Hammer argue that these behaviors were indicative that each member of the group expected to work on a personal task, specifically, answering the question on the worksheet.

Distinguishing Just Math from other e-frames (especially the Worksheet e-frame) requires an analysis of student discourse. Irving et al. analyzed the content of student talk along two axes [8]: "narrow vs. expansive" and "silly vs. serious". "Narrow vs. expansive" focuses on the scope of student discussion, while "silly vs. serious" focuses more on how playfully the students interact while in a Discussion e-frame. Irving et al. found that when students work towards the goal of solving a problem, their talk is most often both narrow and serious.

\section{ANALYSIS}

By combining e-frames with two-axis framing, we identified the Just Math frame. From the perspective of student behavior, Just Math is similar to the Worksheet eframe: a hunched posture, hands busy writing, gaze focused on the page, and discourse that is typically brief. However, applying the two framing axes to the content of students' talk distinguishes Just Math from the Worksheet e-frame. We coded 9 hours of video for student behaviors, discourse, and expectations.

The coding process began by first focusing on coding the HHS data for student behaviors. Two researchers (SFW and LD) independently reviewed approximately 9 hours of HHS data looking for postures, gestures, and gaze patterns consistent with the Worksheet and Just Math e-frames. These researchers negotiated (with each other and, later, with members of the team) the start and stop times associated with what they believed were candidate episodes of Just Math. After negotiation, we found approximately 1.5 hours of candidate HHS data where students exhibited behaviors consistent with the Worksheet or Just Math e-frames.

Having identified candidate episodes using an analysis of student behaviors, we analyzed the content of student talk during these candidate episodes. Episodes where student talk included narrow and serious math-focused check-ins (see Episode 1) and/or expansive and silly comments (see Episode 2) were identified as Just Math. These episodes were discussed and the relevant features negotiated among members of the team.

If there was insufficient evidence of an episode being Just Math from the analysis of the discourse and behaviors then the analysis process switched to expectations, where the coders micro-analyzed the behaviors and discourse before and after candidate Just Math episodes for the groups expectations. The combined analysis for behaviors, discourse and expectations resulted into the identification of approximately 10 minutes of HHS data that could be definitively categorized as Just Math. We believe that, to date, there are unidenitfied episodes of Just Math present within the 1.5 hours of candidate HHS data but there are a number of challenges to definitively identifying episodes of Just Math, which will be discussed later. In the following sections, we discuss the three elements of the Just Math e-frame through examples drawn from our data.

\section{Behaviors}

The behaviors observed while students are in the Just Math e-frame are similar in nature to the behaviors described for the previously discussed Worksheet e-frame. Figure 1 illustrates the posture of the students before, during, and after Just Math. In the left frame, students are just beginning to enter Just Math; they hunch over their respective papers and shift their gaze from the group and its resources to their own papers. In the middle frame, we see the students' hands are busy writing. Some students pause briefly to check in with the group or a personal resource, such as a textbook, and then return to writing. In the right frame, we see that students have left Just Math; they are now sitting upright and looking at each other while discussing their solutions to the problem.

\section{Discourse}

In order to distinguish Just Math from the Worksheet e-frame, we analyzed the students' discourse. First, we noticed that the rate of talk decreases when students are engaged in Just Math. Furthermore, the nature of that talk fell into one of two categories: 


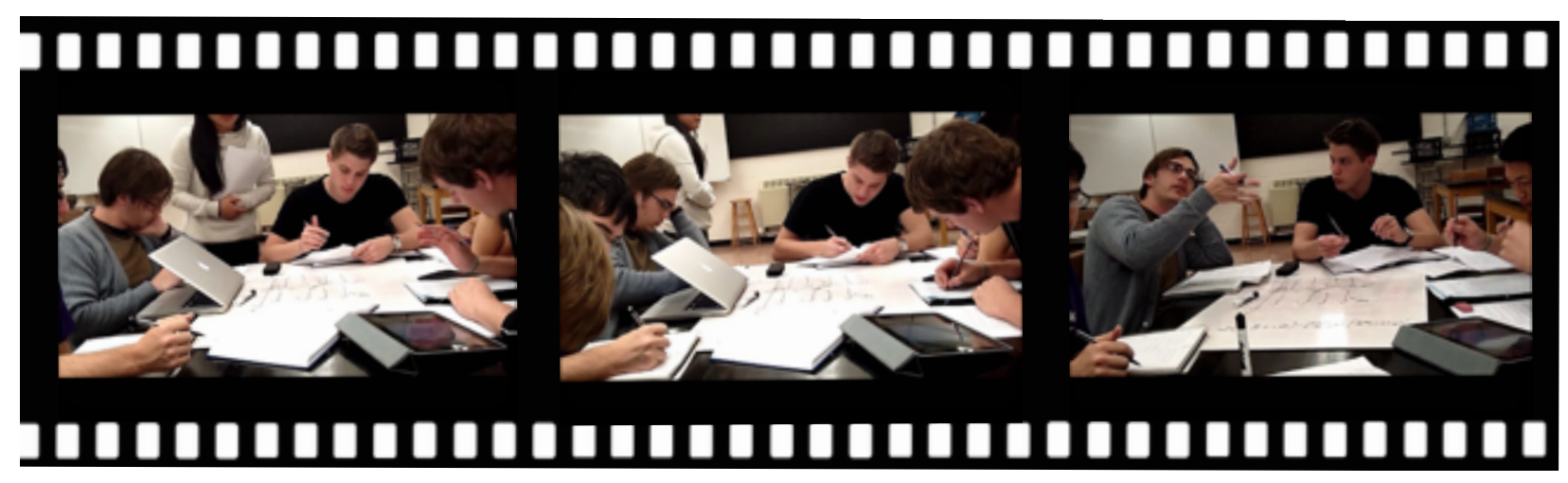

FIGURE 1. Group body language before and during Just Math epistemological frame - Jonah group week 3. In the left frame, students focus is shifting to their own papers after engaging in a group discussion. In the center, we see students exhibiting Just Math behaviors. On the right, students have exited Just Math, and are now discussing their solutions to the problem.

(1) Narrow and serious math-focused check-ins about the problem (e.g., the integral of sine is positive or negative cosine), which are brief in nature. In the following episode, 5 students are sitting around a table, working on a problem to determine if a particular static magnetic field has no curl. Students begin the episode with a discussion about the curl including consulting the front flyleaf of the textbook. Once they begin taking the curl, students focus on writing on their own papers (left frame of Figure 1). After a couple of minutes of writing, a student decides to check-in with his group:

\section{Episode 1}

L: So this is zero because the phi component is constant with respect to $z$, right?

J: Yeah

After this brief exchange the group returns to noncommunicative, paper-focused behavior (middle frame of Figure 1).

(2) Expansive and silly (off-topic) comments while the students are working. This second kind of talk was not reported in Scherr and Hammer's Worksheet e-frame and does not fit in the Joking e-frame from a behavioral perspective. In the following episode, 3 students are working on a problem that is requiring them to take the curl of a magnetic field in cylindrical coordinates. Once the group has entered Just Math, they begin a discussion that is off-topic:

\section{Episode 2}

L: Boom, good job Jonah, you're ahead of me on this

J: Well, I started in the complete wrong spot so...

L: Have you and Ian busted out that telescope yet?

From this introductory question an off-topic conversation about telescopes continues. For the whole exchange, the students continue to focus on their own personal papers, and do not share gaze with each other or attend to their joint whiteboard. All of the students continue to write and process through the remaining math of the problem while concurrently talking about telescopes. Both of these types of talk illustrated are characteristic of the Just Math e-frame.

\section{Expectations}

Group expectations can be inferred from the behaviors and discourse of the group before, during, and after Just Math. The HHS is an environment where the expectation is to obtain a solution to the homework problem. The focus of the discussions prior to entering Just Math is on building a mathematical procedure or representation from the shared resources of the group. This procedure or representation is typically negotiated until the group reaches a point in time where each member is comfortable to proceed with "doing the math." The following episode contains the discussion that occurs prior to the students entering Just Math in Episode 2, which was discussed previously.

\section{Episode 3}

$\mathbf{J}:$ It gives us an equation for B... and I think it's just find like... take the curl of it... but then the next part says suppose that the field decreases as $r^{2}$ instead of $r$, show that the curl is non-zero.

L: Yeah but I mean if you look at the...curl for it in cylindrical coordinates and you can show that... the terms go to zero.

This discussion of the curl and how to take it in cylindrical coordinates continues for a few minutes until the participants are ready to do the math and enter into Just Math. The scaffolding by the students through narrow/serious discussion to a point in the problem where 
they as a group can enter into Just Math is an essential element of our ability to identify the Just Math e-frame and distinguish it from the Worksheet e-frame. Once the rest of the group agrees, Just Math begins. Once the transition into Just Math has occurred, there are several expectations that become apparent and observable through the student's behavior and discourse. The main expectation being that to proceed with the problem they need to "do math". Students also have the expectation that "doing math" is a solitary activity. These expectations are illustrated by the math based check-ins (Episode 1) and the behavioral cues previously discussed. There is also an expectation that being in the Just Math e-frame is not as taxing as the discussion that preceded it and that they can partake in silly off-topic talk while processing through the math (Episode 2). There is also an expectation that it is possible that the scaffolding they have constructed is not mathematically sound and that bids to transition back to a Discussion e-frame are allowed if a complication arises with the math.

\section{DISCUSSION AND CONCLUSIONS}

In this paper, we identified the Just Math e-frame, defined by students' behavior, discourse, and inferred expectations. The Just Math e-frame is strongly related to the Worksheet e-frame [7], but also includes important differences. The behaviors that students engage in are typical of the Worksheet e-frame. Distinctions between the Just Math frame and the Worksheet e-frame are evident based on the content of the group discourse. This includes the possibility of off-topic conversation while processing through the math but more importantly the emphasis on math-oriented discourse to infer shared framing and expectations. Both the check-ins during Just Math and the scaffolding that occurs before transitioning are math centered. When students enter the Just Math eframe one expectation is that in order to proceed with or finish the problem they must do math. This is in contrast to the Worksheet e-frame where students' expectation is that they now need to write down an answer. This difference will affect what students do before they enter the frame and how they proceed when they exit the frame.

Finding the Just Math frame in natural observations presents some methodological challenges. Our analysis is necessarily confined to contexts where students work together in groups and talk about their problem solving. While we believe that individuals may enter Just Math when working alone, our analysis has not uncovered that in a naturalistic setting. Furthermore, even in groups, we are dependent on students' discourse to distinguish it from the Worksheet e-frame. The check-ins that students make during Just Math are an important aspect of our ability to distinguish between it and the Work- sheet e-frame due to the overlapping behaviors between the two. However, check-ins only occur if the students encounter a problem. Encountering a problem does not always occur because the group may have scaffolded the Just Math frame adequately or may not need to check on a more trivial element of the math needed to solve the problem. Hence, the amount of data that we identified as Just Math is likely the lower measure for this data set. This point merely highlights the need to look at the e-frames from which students move into Just Math so that we can better identify when students are doing math. In the future we will characterize the pre and post Just Math behaviors, discourse, and expectations in greater detail in order to build up a library of before, during, and after indicators of Just Math.

The Just Math e-frame is, by itself, of limited importance but it is the future work that its identification facilitates that makes its identification valuable. Transitions into and out of Just Math are a fruitful area of future research. Students' sense-making and scaffolding to bring their group to the point where it can enter Just Math is especially interesting, because research on how students use mathematics in group settings is sparse. Different amounts of scaffolding may need to be provided by and for different group members in order to result in a common negotiated Just Math-ready group state. Identifying Just Math allows us to examine this negotiation and scaffolding process and the individual transitions into and out of Just Math. The study of this combination of math related elements should provide greater insight into how students use math in physics contexts.

The authors would like to thank Ying Chen, who identified one of the examples of Just Math presented here and staffed the HHS for the course we observed. We also thank the members of PERL@MSU for their useful comments and suggestions on a draft of the manuscript.

\section{REFERENCES}

1. G. Zhu, and C. Singh, Am. J. Phys. 80, 252 (2012).

2. W. M. Christensen, and J. R. Thompson, Phys. Rev. ST-PER 8, 023101 (2012).

3. B. R. Wilcox, M. D. Caballero, D. A. Rehn, and S. J. Pollock, Phys. Rev. ST-PER 9, 020119 (2013).

4. D. Hammer, Am. J. Phys. 68, S52 (2000).

5. E. C. Sayre, and M. C. Wittmann, Phys. Rev. ST-PER 4, 020105 (2008).

6. D. Hu, and N. S. Rebello, Phys. Rev. ST-PER 9, 020108 (2013).

7. R. E. Scherr, and D. Hammer, Cognition and Instruction 27, 147-174 (2009).

8. P. W. Irving, M. S. Martinuk, and E. C. Sayre, Phys. Rev. ST Phys. Educ. Res. 9, 010111 (2013). 